\title{
Assessing and comparing the innovativeness and creative climate of firms.
}

\begin{abstract}
The innovativeness of eight manufacturing firms (or four matched pairs of firms) in Malaysia was measured and compared using certain criteria. Initially, we selected the four matched pairs of firms (each pair is comprise of a more innovative and a less innovative firm) from various manufacturing sectors based on whether or not the firms in each pair have implemented computerized manufacturing systems. Further assessments of the innovativeness of the firms were based on: changes/continuous improvements carried out; introduction and implementation of technologies (machines, processes, etc.); interactions of the firms with their external environments; and number of training programs organized by the firms to stimulate innovation and creativity among its employees. In addition to assessing the innovativeness of the firms, the creative climate questionnaire (CCQ) developed by Ekvall et al. was utilized to assess their creative climates. As expected, the results obtained demonstrate that the more innovative firms were found to have introduced more computerized systems and incremental and technological changes, to have more interactions with their external environments, to have organized more training programs aimed at encouraging creativity and innovation, and to have more creative climates when compared to the less innovative ones. The results of this study indicate that in order for a firm to be innovative, it needs to continuously implement many types of changes and technologies in response to environmental needs. Furthermore, the results also confirm in a different culture (i.e. a developing country) earlier Scandinavian studies which have been used to justify the international use of the Ekvall's CCQ. However, it appears that, although the CCQ is a good predictor of higher and lower innovative performance of firms, the instrument on its own is unable to indicate the extent of innovative strengths/weaknesses without using additional criteria or benchmarking.
\end{abstract}

Keyword: Creative climate; Innovation; Innovativeness; Creative climate assessments. 